\title{
A BOUND FOR THE MEAN VALUE OF A FUNCTION
}

\author{
J. ERNEST WILKINS, JR.
}

Let $f(t)$ be a bounded measurable function defined when $0 \leqq t \leqq \pi$. The Fourier sine series associated with $f(t)$ is

$$
\sum_{n=1}^{\infty} b_{n} \sin n t, \quad b_{n}=\frac{2}{\pi} \int_{0}^{\pi} f(t) \sin n t d t .
$$

We shall be interested in this paper in establishing a bound for the mean value ${ }^{1}$

$$
a=\frac{1}{\pi} \int_{0}^{\pi} f(t) d t
$$

when $f(t)$ is such that one of the coefficients $b_{n}$ vanishes.

We can suppose without essential loss of generality that $|f(t)| \leqq 1$. Since $b_{2 n}=0$ whenever $f(t)$ is constant, it is clear that the only conclusion on $a$ that can be drawn from the inequality $|f(t)| \leqq 1$ and the equality $b_{2 n}=0$ is that $|a| \leqq 1$, and this conclusion is valid whether $b_{2 n}$ vanishes or not. Hence we shall restrict attention to $b_{2 n+1}$. For the same reason we shall not discuss the vanishing of the coefficient

$$
a_{n}=\frac{2}{\pi} \int_{0}^{\pi} f(t) \cos n t d t
$$

of the Fourier cosine series of $f(t)$.

Suppose that $b_{2 n+1}=0$. Define a positive number $y$ by the equation $y=\sin \left[\sec ^{-1}(2 n+2)\right]$ where the $\sec ^{-1}$ lies between 0 and $\pi / 2$. Let $E$ be the sum of the intervals

$$
\frac{2 p \pi+\sin ^{-1} y}{2 n+1} \leqq t \leqq \frac{(2 p+1) \pi-\sin ^{-1} y}{2 n+1} \quad(p=0,1, \cdots, n),
$$

where the $\sin ^{-1}$ lies between 0 and $\pi / 2$. Then it is clear that

$$
\begin{array}{lr}
\sin (2 n+1) t \geqq y & \text { if } t \text { is in } E, \\
\sin (2 n+1) t<y & \text { if } t \text { is not in } E .
\end{array}
$$

Received by the editors February 11, 1948, and, in revised form, June 14, 1948.

${ }^{1}$ The importance of the concept of mean value in the study of Fourier series can be seen by consulting Bohr [1, pp. 7-29]. Numbers in brackets refer to the bibliography at the end of the paper. 
Now let $f_{0}(t)=-1$ if $t$ is in $E$ and $f_{0}(t)=+1$ if $t$ is not in $E$. It follows from the definitions of $y$ and $E$ that

$$
\int_{0}^{\pi} f_{0}(t) \sin (2 n+1) t d t=0,
$$

and that the mean value of $f_{0}(t)$ is

$$
\begin{aligned}
a_{0} & =1-(2 / \pi) \text { meas } E \\
& =[4(n+1) /(2 n+1) \pi] \sec ^{-1}(2 n+2)-1 /(2 n+1) .
\end{aligned}
$$

We shall now prove that if $f(t)$ is an arbitrary real-valued measurable function such that $|f(t)| \leqq 1$ and $b_{2 n+1}=0$, then $|a| \leqq a_{0}$. Let $g(t)=f(t)-f_{0}(t)$. Then $0 \leqq g(t)$ on $E$ and $0 \geqq g(t)$ on the complement $c E$ of $E$. By virtue of relations (1) we conclude that

$$
\begin{aligned}
& \int_{E} g(t) \sin (2 n+1) t d t \geqq y \int_{E} g(t) d t, \\
& \int_{c E} g(t) \sin (2 n+1) t d t \geqq y \int_{c E} g(t) d t .
\end{aligned}
$$

Adding and remembering that $b_{2 n+1}=0$ for both $f$ and $f_{0}$ we see that

$$
0 \geqq y \int_{0}^{\pi} g(t) d t,
$$

with equality if and only if $g(t)=0$ almost everywhere. Since $y>0$, we have that

$$
\int_{0}^{\pi} f(t) d t \leqq \int_{0}^{\pi} f_{0}(t) d t
$$

with equality if and only if $f(t)=f_{0}(t)$ almost everywhere.

Now let $h(t)=f(t)+f_{0}(t)$. Then $0 \geqq h(t)$ on $E, 0 \leqq h(t)$ on $c E$, and so

$$
\begin{aligned}
\int_{E} h(t) \sin (2 n+1) t d t & \leqq y \int_{E} h(t) d t \\
\int_{c E} h(t) \sin (2 n+1) t d t & \leqq y \int_{c E} h(t) d t \\
0 & \leqq y \int_{0}^{\pi} h(t) d t \\
-\int_{0}^{\pi} f(t) d t & \leqq \int_{0}^{\pi} f_{0}(t) d t
\end{aligned}
$$


with equality if and only if $f(t)=-f_{0}(t)$ almost everywhere. Combining the inequalities (2) and (3) we conclude that when $f(t)$ is a real-valued measurable function such that $|f(t)| \leqq 1$ and $b_{2 n+1}=0$, then

$$
|a|=\left|\frac{1}{\pi} \int_{0}^{\pi} f(t) d t\right| \leqq \frac{4(n+1)}{(2 n+1) \pi} \sec ^{-1}(2 n+2)-\frac{1}{2 n+1},
$$

with equality if and only if $f(t)= \pm f_{0}(t)$ almost everywhere.

In particular, if $b_{1}=0$, then $|a| \leqq 1 / 3=.3333$, while if $b_{3}=0$, then $|a| \leqq .7855$. The right-hand side of the inequality (4) approaches unity as $n$ approaches infinity.

This conclusion may be extended to complex functions $f(t)$ as follows. Let $f(t)=f_{1}(t)+i f_{2}(t)$, where $f_{1}(t)$ and $f_{2}(t)$ are real. There exist real numbers $x$ and $y$ such that

$$
x^{2}+y^{2}=1, \quad x \int_{0}^{\pi} f_{2}(t) d t+y \int_{0}^{\pi} f_{1}(t) d t=0 .
$$

Hence it is true that the mean value of $f(t)$ has the same absolute value as the mean value of the real function $x f_{1}(t)-y f_{2}(t)$. This real function has a Fourier coefficient $b_{2 n+1}$ equal to zero since this is true for both $f_{1}(t)$ and $f_{2}(t)$ and is bounded by one since $f(t)$ is and $x^{2}+y^{2}$ $=1$. Since the inequality $(4)$ is valid for $x f_{1}-y f_{2}$, it is therefore true for $f(t)$. Moreover since equality for $x f_{1}-y f_{2}$ implies that $x f_{1}-y f_{2}$ $= \pm f_{0}(t)$, equality for $f(t)$ implies that $f(t)=c f_{0}(t)$ where $c$ is a constant of absolute value unity.

\section{BIBLIOGRAPHY}

1. H. Bohr, Almost periodic functions, New York, Chelsea, 1947.

American Optical Company 